\title{
Hausstaubmilben-Allergie
}

\section{Atemnot durch kleine Plagegeister}

\author{
Leiden Sie ständig unter einer verstopften Nase, tränenden Augen, wo- \\ möglich sogar unter Atemnot? Hinter solchen Beschwerden kann eine \\ Hausstaubmilben-Allergie stecken. Hellhörig werden sollten Sie auch \\ dann, wenn die Symptome sich in der Heizperiode verschlimmern. Pro- \\ fessor Karl-Christian Bergmann vom Allergiezentrum der Berliner Chari- \\ té erläutert die Hintergründe.
}

? Herr Professor Bergmann, welche Rolle spielen Hausstaubmilben bei der Entstehung von Allergien?

Bergmann: Hausstaubmilben sind nach den Pollen die zweitwichtigsten Auslöser von Allergien. Sie führen zu einem meist chronischen allergischen Schnupfen und Bindehautentzündungen der Augen. Im schlimmsten Fall können sie auch allergisches Asthma auslösen.

? Wo lauern die Milben? Begegnen sie uns auch außerhalb der eigenen vier Wände?

Bergmann: Früher hat man die Hausstaubmilben meist nur im Schlafzimmer und Bett vermutet, auch in den Teppichen dort. In letzter Zeit mehren sich Befunde dafür, dass sie auch in Verkehrsmitteln, im Büro oder auch in den anderen Räumen der Wohnung - übrigens auch in Kellern - vorhanden sind. In Krankenhäusern, wo das Bettzeug ja regelmäßig ausgetauscht wird und Oberflä- chen täglich gereinigt werden, spielen solche Allergien hingegen keine Rolle.

? Bei welchen Symptomen sollte man denn an eine Milbenallergie denken?

Bergmann: Wer ständig eine verstopfte Nase hat und immer wieder mal Niesen muss und dies besonders in Innenräumen, Auto, Büro oder im Kino, bei dem kann es sich um einen Milbenschnupfen handeln. Es muss nicht unbedingt jucken, wie das bei einem Heuschnupfen der Fall ist.

? Was heißt das für den Betroffenen wann sollte er sich einer Behandlung unterziehen?

Bergmann: Wenn die Nase verstopft ist, kann man oft nicht mehr richtig Arbeiten oder Denken, auch der Schlaf ist häufig gestört. Also, ab zum Allergologen oder HNO-Arzt und in einem Hauttest prüfen, ob man Antikörper gegen Milben in der Haut (Allergietest) oder im Blut hat. Dann am

\section{Hausstaubmilben}

Die Spinnentierchen ernähren sich von abgestorbenen Hautschuppen und sind nur $0,1-0,4 \mathrm{~mm}$ groß. Mit dem bloßen Auge sind sie also praktisch nicht erkennbar. Besonders gut vermehren sich Hausstaubmilben bei einer Raumfeuchtigkeit von $75 \%$ und einer Temperatur von $15^{\circ}$ Celsius. Daher sind sie in erster Linie in Schlafzimmern sehr verbreitet. Rund 16\% aller Erwachsenen und 22\% der Kinder in Deutschland sind gegen Hausstaubmilben sensibilisiert. Als Allergene wirken dabei vor allem Eiweißstoffe aus dem Kot der Milben.

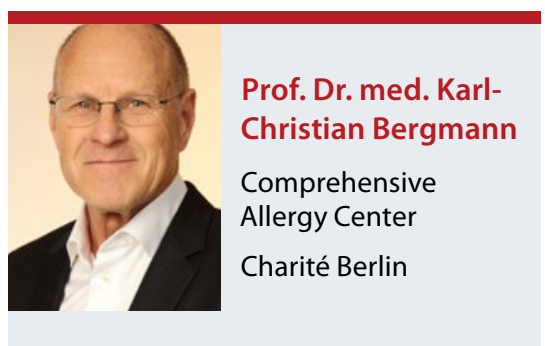

besten mit einem nasalen Provokationstest kontrollieren, ob das Milbenallergen auch die Nasenbeschwerden auslöst.

\section{? Und dann?}

Bergmann: Wenn das der Fall ist, kann die von der Charité Berlin und der Medizinischen Universität Wien entwickelte kostenlose App „Milbencheck" weiterhelfen (www. milbencheck.de). Prüfen und dokumentieren Sie, wo die stärksten Beschwerden auftreten und wo Milben sein könnten. Danach besprechen Sie mit dem Arzt die Medikamente. Reichen anti-allergische Tabletten oder sollte doch besser eine Immuntherapie erfolgen? Letzteres ist sicher auf Dauer besser - zumal es jetzt in Deutschland neben den üblichen Spritzen auch Milbentabletten oder -tropfen gibt, die sehr gut verträglich und wirksam sind!

? Was können Allergiker noch tun, um ihre Beschwerden in Schach zu halten?

Bergmann: Grundsätzlich gilt: Vermeiden Sie den Kontakt mit den Allergenen, auch wenn es praktisch unmöglich ist, Milben komplett aus dem häuslichen Umfeld zu verbannen. Mit einigen Basismaßnahmen lässt sich die Milbenbelastung aber spürbar senken: So sollten Matratzen und Bettzeug mit milbenundurchlässigen Bezügen versehen werden. Wichtig ist es auch, drei- bis viermal täglich kräftig zu lüften. Sorgen Sie dafür, dass die Luftfeuchtigkeit unter $50 \%$ liegt. Auch auf Staubfänger wie Teppiche, schwere Vorhänge etc. sollten Hausstaubmilben-Allergiker möglichst verzichten. Überhaupt: Bevorzugen Sie pflegeleichte Materialien und Beläge, die feucht gewischt werden können. 